\title{
Computation of a Controlled Store Separation from a Cavity
}

\author{
Christopher A. Atwood, \\ MCAT Institute. NASA Ames Research Center, \\ Moffett Field, California 94035-1000 \\ Extended abstract submitted for the \\ AIAA 32nd Aerospace Sciences Meeting and Exhibit \\ January 10-13, 1994, Reno, NV \\ May 14, 1993
}

\begin{abstract}
Coupling of the Reynolds-averaged Navier-Stokes equations, rigid-body dynamics, and a pitch attitude control law is demonstrated in two- and three-dimensions. The application problem was the separation of a canard-controlled store from an open-flow rectangular cavity bay at a freestream Mach number of 1.2. The transient flowfield was computed using a diagonal scheme in an overset mesh framework, with the resultant aerodynamic loads used as the forcing functions in the nonlinear dynamics equations. The proportional and rate gyro sensitivities were computed apriori using pole placement techniques for the linearized dynamical equations. These fixed gain values were used in the controller for the nonlinear simulation. Reasonable comparison between the full and linearized equations for a perturbed two-dimensional missile was found. Also in two-dimensions, a controlled store was found to possess improved separation characteristics over a canard-fixed store. In three-dimensions, trajectory comparisons with wind-tunnel data for the canard-fixed case will be made. In addition, it will be determined if a canard-controlled store is an effective means of improving cavity store separation characteristics.
\end{abstract}

\section{Nomenclature}

$\begin{array}{ll}C_{m} & \text { coefficient of pitching moment } \\ D O F & \text { degree of freedom } \\ I & \text { body inertia } \\ K_{a} & \text { proportional gain } \\ K_{r} & \text { rate gyro sensitivity } \\ L & \text { characteristic length } \\ m & \text { body mass or stage number } \\ M & \text { Mach number } \\ q & \text { dynamic pressure } \\ Q & \text { vector of dependent variables } \\ R e & \text { Reynolds number } \\ s & \text { Laplace operator }\end{array}$

* Research Scientist, MCAT Institute. Member AIAA. 


$\begin{array}{ll}t & \text { time } \\ T & \text { absolute temperatume } \\ u, u, u & \text { Cartesian velocity components } \\ x, y, z & \text { Cartesian physical space } \\ \alpha & \text { angle of attack } \\ \delta & \text { effector deflection angle } \\ \rho & \text { density } \\ \sigma & \text { shear layer spreading rate parameter } \\ \tau & \text { canard servo characteristic time } \\ \theta & \text { body attitude } \\ \omega & \text { vorticity } \\ (\text { ) } & \text { time derivative } \\ \text { Superscripts } \\ n & \text { time step } \\ \text { Subscripts } \\ c & \text { commanded, canard } \\ t & \text { tail } \\ \infty & \text { freestream quantity }\end{array}$

\section{Introduction}

The design of high-performance aircraft has in the past typically been compartmentalized by discipline. most commonly structures, fluids, and controls. However, as performance demands escalate. aircraft systems have become increasingly interrelated. ${ }^{1,2}$ Therefore, there is a need to investigate the optimization of the aircraft in its entirety, not simply by evaluation of its sub-systems.

This type of multidisciplinary analysis is currently accomplished with simplified physical models. such as panel flow and modal structural codes. However, in critical regions of the flight envelope these linear methods can fail, leading to the necessity for higher-order models. ${ }^{3.4}$ Obviously. this increased physical fidelity comes with a high computational price, and hence fewer design cycles are permitted as compared to the linear methods.

Providing a capability for the simulation of the nonlinear interaction of fluids and rigid body motion will be useful in several ways. First, the simulation could be used to validate the implementation of a control law derived using conventionally determined sensitivity coefficients. Although one must be wary of results obtained numerically, simplifications used to compute these force and moment derivatives will not be present in a Navier-Stokes simulation. Second, the coupled simulations could be used to develop a control law where nonlinear effects are important, using the computed aerodynamic forces and moments instead of tabulated empirical relationships. ${ }^{5}$ Hence, computational design and prototyping of the aircraft control system offers the capability of reducing aircraft design cycle cost and enhancing safety as well as improving performance.

The effort documented here begins to address the interaction of the disciplines of fluids. rigid body dynamics, and controls in nonlinear flight regimes. In order to assess the accuracy of these initial computations. a problem which could be compared against analytic and experimental results was chosen: the cavity store separation problem. Dix and Dobson ${ }^{6}{ }^{6}$ recent experimental study of the separation of stores from cavity bays will be used as a basis for comparison. These wind-tunnel tests determined the trajectory of an uncontrolled missile from a rectangular cavity and will be used to validate the canard-fixed computation.

Previous computational efforts have shown that the component problems of cavity flows ${ }^{\bar{\tau}, 8,9}$ and uncontrolled store separation ${ }^{10,11}$ can be solved with reasonable accuracy using overset grid methods. It should be noted that inviscid solutions to the cavity store separation problem, with bodies in relative motion, have been obtained on tetrahedral meshes. ${ }^{12}$ However, although these unstructured methods are 


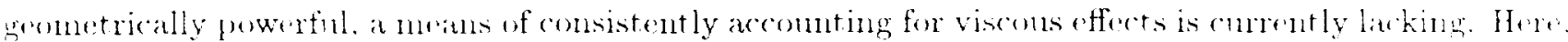

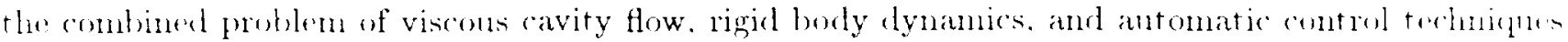
is addressed in a general manner using the overset mesh franework.

The following sections discuss the approach nsed to solve the coupled system and the lesults obtained for several two- and three-dimensional cases. Comparisons of numerical results are made against linearized or experimental results where available.

\section{Approach}

The procedure used to solve these coupled problems is shown schematically in Fig. 1. The process

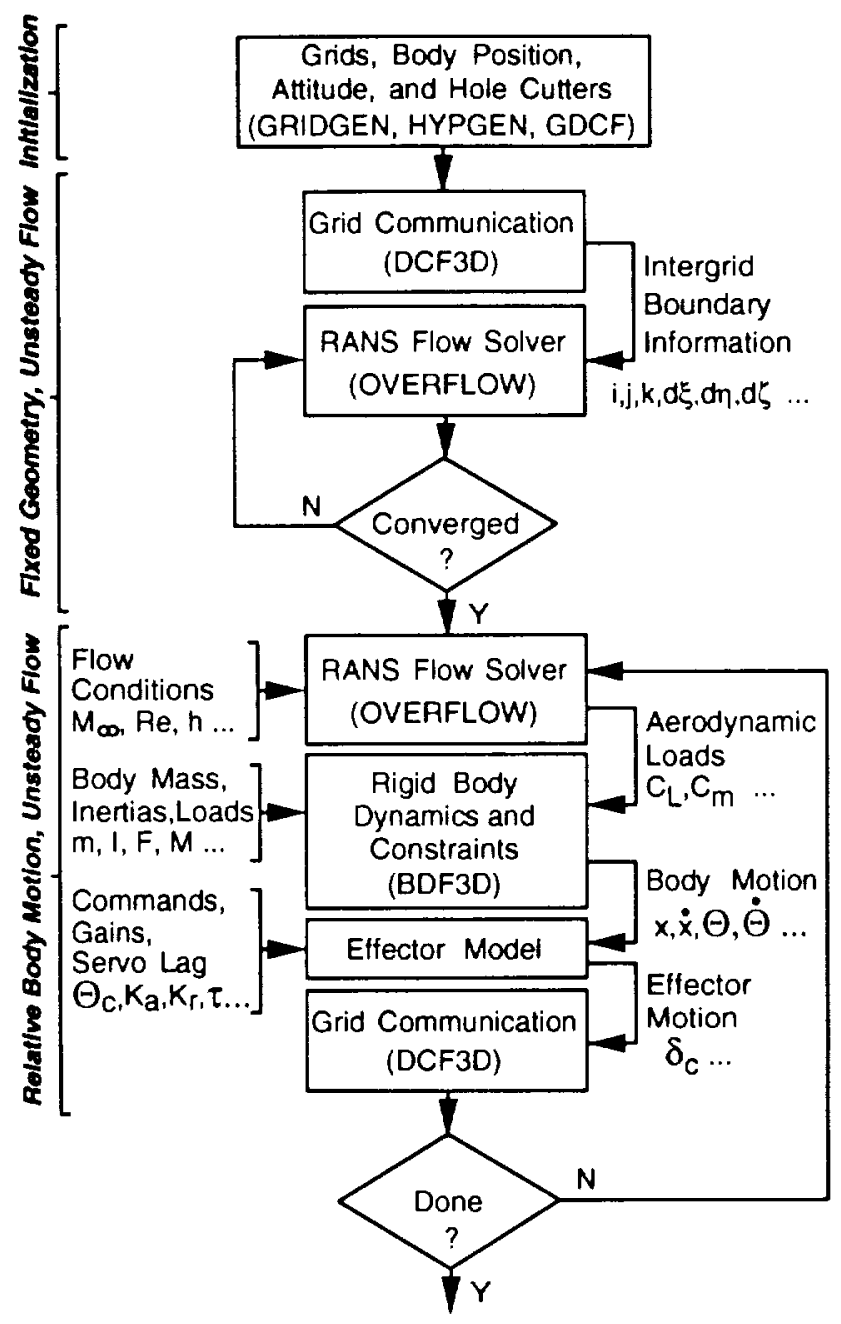

Fig. 1: Overall coupled system approach

begins with the generation of the blocked mesh system while holding the relative position of the geometry in the initial position. After establishing the initial grid connectivity information, the solution of the flowfield can begin. Obviously, depending on the problem being addressed. this fixed grid solution may be steady or possess a bounded envelope. When this fixed-geometry flow solution is satisfactory. the motion of the body commences.

The integrated aerodynamic loads acting on the body are provided to the 6-DOF portion of the code along with the body state vector. Using this information, the body position and attitude is then integrated one time step, and kinematic constraints are applied. The controller also uses the body state to compute new effector settings, which are then applied to all relevant grids. Finally. since the grids 


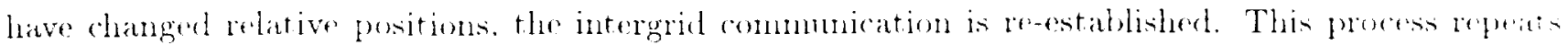
for each time step until the simulation is complete. A detailed description of the conponent processes is given below.

\section{Flow Solver}

The fluid field was computed via the Reynolds-averaged Navier-Stokes (RANS) equations using the diagonal scheme of Pulliam and Chansse ${ }^{13}$ implemented in the overset grid framework of Benek. Buning, and Steger. ${ }^{14}$ The equations were integrated through Euler implicit time marching and second-order spatial differencing with viscous wall conditions specified as no-slip, zero normal pressure gradient. and adiabatic. Information transfer across overset mesh boundaries was implemented using trilinear interpolation of the dependent variable vector, $Q=[\rho, \rho u, \rho v, \rho w, e]^{T}$. The flow solver cost is $13 / \iota s / \mathrm{cell} / \mathrm{step}$ on a Cray Y-MP.

\section{Turbulence Model}

In these computations, the slowly time-varying component of the flow is resolved, while rapid fluctuations are modelled. The algebraic turbulence model of Baldwin and Lomax. ${ }^{15}$ as implemented by Buning, ${ }^{16}$ is described below using a flow in the $(x, y)$ plane.

The wall-bounded flows use the original Baldwin-Lomax model, with the addition of a variable $F_{\max }$ cutoff. The grids are chosen such that a unique wall distance is readily available.

The cavity aperture spanning shear layer uses an eddy viscosity developed using $F(y)=y|\omega|$. as suggested by Baldwin and Lomax ${ }^{15}$ for wake regions. This results in

$$
\begin{aligned}
F_{w a k e} & =C_{u^{\prime} k} \frac{y_{\max } u_{\text {dif }}^{2}}{F_{\text {max }}} \\
& =C_{w \cdot k}\left(\frac{u_{\text {dif }}}{|\omega|_{\max }}\right)^{2}|\omega|_{\max } \\
& \propto \ell^{2}|\omega|
\end{aligned}
$$

where specification of $C_{w k}$ is discussed below and the velocity difference is modified to be half the total velocity difference between the streams in the specified shear layer region

$$
u_{d i f}=\sqrt{\left(u^{2}+v^{2}\right)}{ }_{\max }-\sqrt{\left(u^{2}+v^{2}\right)}{ }_{|\omega|_{\max }}
$$

The free shear layer model is now given by

$$
\mu_{t}=\rho K C_{c p} C_{u k k} \frac{u_{d i f}^{2}}{|\omega|_{\text {max }}}
$$

after dropping the Klebanoff intermittency function. Unmodified model constants $K=0.0168$ and $C_{c p}=1.6$ are used.

The magnitude of the eddy viscosity in the free shear layer model can be altered with $C_{u k}$. Estimation of the proper value of $C_{u k k}$ begins by using Görtler's shear layer solution:

$$
\begin{aligned}
& u=\frac{u_{1}+u_{2}}{2}\left[1+\frac{u_{2}-u_{1}}{u_{2}+u_{1}} \operatorname{erf}(\xi)\right] \\
& \operatorname{erf}(\xi)=\frac{2}{\sqrt{\pi}} \int_{0}^{x} e^{-\xi^{2}} d \xi, \xi=\frac{\sigma y}{x}
\end{aligned}
$$

where $u_{1}$ and $u_{2}$ are the velocities of the slow and fast streams and $\xi$ is the similarity coordinate. The spreading parameter $\sigma$ is inversely related to the spreading rate, $d b / d x$, where $b$ is a measure of the 


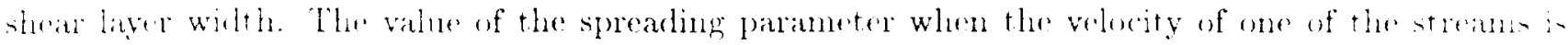
zen is $\sigma_{11}$.

Görtlers solution can be used to determine the maximum vorticity magnitude as follows:

$$
\begin{aligned}
|\omega| & =\left|\frac{\partial u}{\partial y}\right|=\left|\frac{\partial u}{\partial \xi} \frac{\partial \xi}{\partial y}\right| \\
& =\left|\frac{\sigma}{x \sqrt{\pi}} \Delta u e^{-\xi^{2}}\right| \cdot \Delta u=\left(u_{2}-u_{1}\right) \\
|\omega|_{\text {max }} & =\frac{\sigma}{x \sqrt{\pi}} \Delta u
\end{aligned}
$$

Now, using Prandtl's mixing length assumption and scaling laws for jet boundaries. eddy viscosity can also be expressed as

$$
\begin{aligned}
\mu_{t} & \propto \rho \ell^{2}|\omega| \\
& \propto \rho \ell^{2} \frac{\Delta u}{b} \\
& =K_{0} \rho b \Delta u
\end{aligned}
$$

where $K_{0}=\frac{r}{4 b \sigma_{0}^{2}}$.

Setting Eqs. 1 and 2 equal results in

$$
C_{w k}=\left(\sigma_{0} K C_{c p} \sqrt{\pi}\right)^{-1}
$$

and only $\sigma_{0}$ remains to be specified. Empirical estimates of $\sigma_{0}$ range from 9.0 to $13.5 .{ }^{9}$ For this series of cavity flow efforts $\sigma_{0}$ was set to 11.0 , resulting in a value of $C_{w k}=1.91$.

\section{Grid Generation and Communication}

Computation of the loads generated by cavity flows requires accurate representation of the geometry as well as the flowfield. Typically, a significant effort in grid generation is required before flow analysis can begin. Since matching zone faces are not required for the overset method used here, recent advances in algebraic ${ }^{17}$ and hyperbolic ${ }^{18}$ methods can be used. Hyperbolic grid generation, which gives good spacing and orthogonality control. was used for the wall-bounded regions, while algebraic grids were used in shear flow regions. Advantages of this type of grid system include straightforward specification of the turbulent regions and allowance for independent refinement of each zone. This topology also permits the re-use of meshes for configuration studies.

Exchange of flow information is accomplished using a domain connectivity function, with the donorreceiver relationship established at each time step using an efficient technique. ${ }^{19}$ Although the cost of re-establishing intergrid communication is problem dependent, the computational expense is generally half of that used by the diagonalized flow solver. The initial location for the hole-cutters was specified using a graphical interface, ${ }^{20}$ after which the movement of the grids and hole cutters were updated altomatically. An example of the overset mesh topology used is shown in Fig. 2. which shows the configuration at an instant in the separation process.

\section{Kinematics and Rigid Body Dynamics}

Although details of the six-DOF dynamical equations can be found elsewhere, ${ }^{21}$ briefly the rotational dynamics is described by Euler's equations of motion which aligns the $x y z$ coordinates with the body

principal axes at the center of gravity. For instance, for a rotation $\theta$ about an axis $\lambda$. Euler parameters can be specified as

$$
\epsilon=\left[\lambda_{1} \sin \frac{\theta}{2}, \lambda_{2} \sin \frac{\theta}{2}, \lambda_{3} \sin \frac{\theta}{2}, \cos \frac{\theta}{2}\right]^{T}
$$




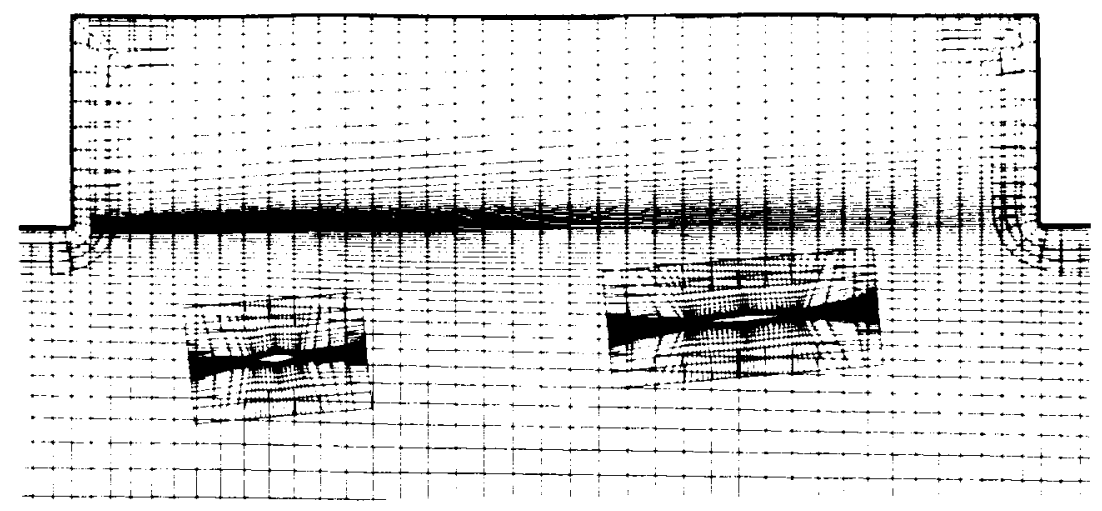

Fig. 2: Coarsened grids after release

These Euler parameters, integrated according to the rotational body dynamics, are updated and stored for each grid. ${ }^{10,19}$ Kinematic constraints can be imposed during the ejection process or for restrictedDOF simulations. In addition, the assumption of rigid-body dynamics eliminates the need to store the component grids for all time, since the Euler parameters may be used to compute grid attitude from the initial position.

Rotating effectors were implemented by summing the relative commanded effector and body angular velocities, $\omega$. Integration of $\omega$ gives the proper effector attitude relative to the initial grid positions. The hinge line location is updated according to the attitude of the body. Storage of the hinge line and Euler parameters associated with each grid allows nesting of parent-child bodies to an arbitrary level without modification to the grid communication and support software.

\section{Pitch Attitude Control Law}

The fourth-order system shown in Fig. 3 was used as the system model for both the two- and

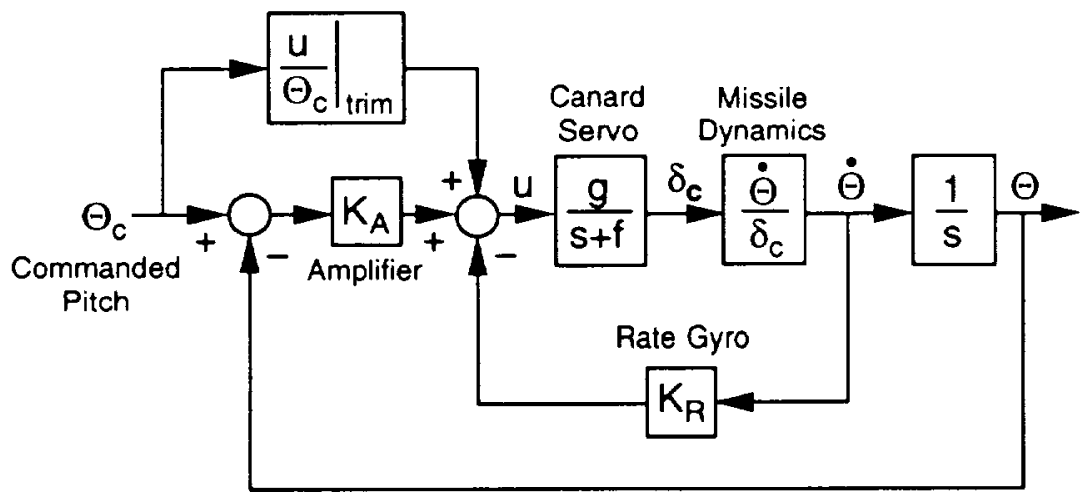

Fig. 3: 2-D missile: Block diagram

three-dimensional missile cases. In Fig. 3 the plant and servo can be expanded as

$$
\frac{\dot{\theta}}{\delta_{c}}=\frac{d s+e}{a s^{2}+b s+c} ; f=\frac{1}{\tau}
$$

where the servo time constant was taken as $\tau=\frac{1}{i 5} \mathrm{~s}$. The plant coefficients were determined from the governing equation; they are composed of geometric and flow information, as well as the stability derivatives. The stability derivatives were determined from linearized supersonic airfoil theory in the two-dimensional cases and from direct computation in three-dimensions. Pole placement techniques and linearized system time response were used to determine the proportional. $K_{a}$, and the rate gyro. $K_{r}$, sensitivities. 


\section{Results and Discussion}

The method drescribed above wibs applied in two-dimensions to a perturbed one degree-of-freerdon case and a three degree-of-freedom cavity store separation process. The three-dimemsional results arr in progress.

\section{One Degree-of-Freedom Simulation}

In order to provide some measure of validation, a two-dimensional, 1-DOF simulation was implemented at a flight altitide of 45,000 ft. Comparison of the linearized and the coupled nonlinear system response for a small perturbation allowed assessment of the basic methodology.

\section{Linearized Dynamics}

The equation of motion, $M_{y}=I_{y y} \ddot{\theta}$, after neglecting the pitch-damping term, is

$$
M_{y}=q_{\infty}\left\{\left[\left(C_{l_{\alpha}} S d\right)_{c}-\left(C_{l_{\alpha}} S d\right)_{t}\right] \theta+\left(C_{l_{\alpha}} S d \delta\right)_{c}\right\}
$$

where the body was pinned at the location of the center of gravity for this analysis.

Expressing the system in a state variable representation, with $\dot{x}=\left[\theta, \dot{\theta}, \ddot{\theta}, \delta_{c}\right]^{T}$. then the open loop equation can be expressed as

$$
\dot{x}=\left[\begin{array}{cccc}
0 & 1 & 0 & 0 \\
0 & 0 & 1 & 0 \\
0 & -\frac{c}{a} & -\frac{b}{a} & \frac{d}{a} \\
0 & 0 & 0 & -f
\end{array}\right] x+\left[\begin{array}{l}
0 \\
0 \\
0 \\
g
\end{array}\right] u
$$

where

$$
\dot{x}=A x+B u ; y=C x ; u=Q x+R r
$$

The closed loop equation is expressed as

$$
\begin{gathered}
\dot{x}=A x+B[Q x+R r]=[A+b Q] x+K_{a} b r \\
A+b Q=\left[\begin{array}{cccc}
0 & 1 & 0 & 0 \\
0 & 0 & 1 & 0 \\
0 & -\frac{c}{a} & -\frac{b}{a} & \frac{d}{a} \\
-\frac{e g}{d} K_{a} & -g\left(K_{a}+\frac{e}{d} K_{r}\right) & -g K_{r} & -f
\end{array}\right] \\
K_{a} b=\left[0,0,0,\left(K_{a}+K_{t}\right) g\right]^{T}
\end{gathered}
$$

The state can be computed using Euler explicit integration

$$
x^{n+1}=\Delta t\left[\left(A+b Q+\frac{I}{\Delta t}\right) x^{n}+K_{a} b r^{n}\right]: y^{n+1}=C x^{n+1}
$$

The gain levels. $K_{a}=1.2$ and $K_{r}=0.06 \mathrm{~s}$, were computed using conventional root locus methods. These gains were input into a linearized one degree-of-freedom dynamics routine to verify implementation of the control law. The result of the linearized analysis is shown in Fig. 4, which shows a slightly divergent envelope for the canard-fixed case, and damped behavior for the closed-loop system. Note that a canard deflection limiter was applied to represent stalling of the airfoil. 


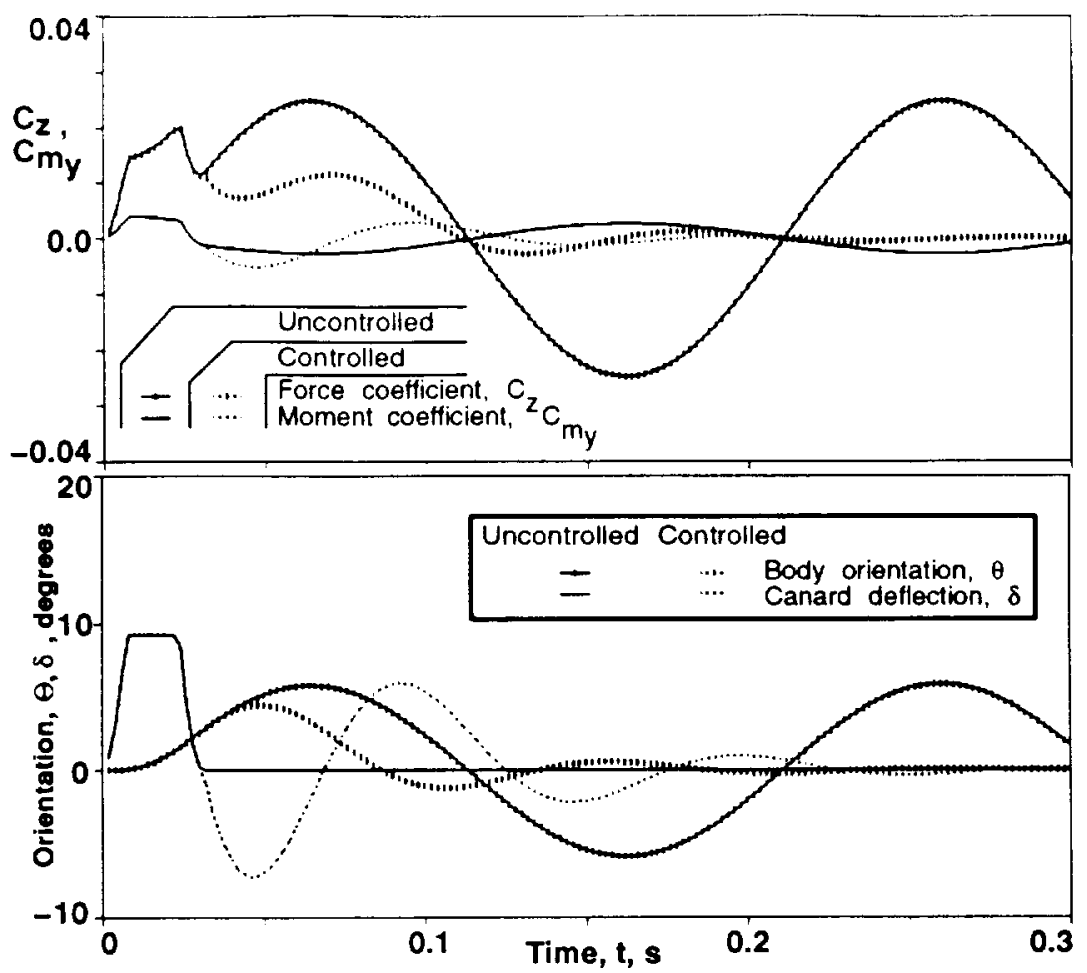

Fig. 4: Linearized 2-D missile: body orientation and canard deflection time histories for both uncontrolled and controlled cases

\section{Nonlinear Coupled Simulation}

The control law developed above was also implemented into the code which coupled the RANS equations with rigid-body dynamics. From Fig. 3. the control law can be expressed as

$$
\delta_{c}=\frac{g}{s+f}\left[K_{a}\left(\theta_{c}-\theta\right)-K_{r} \dot{\theta}+K_{t} \theta_{c}\right]
$$

and integrated using Euler explicit integration

$$
\delta_{c}^{n+1}=\frac{g \Delta t}{f \Delta t+1}\left\{\left[K_{a}\left(\theta_{c}-\theta^{n}\right)-K_{r} \dot{\theta}^{n}+K_{t} \theta_{c}\right]+\delta_{c}^{n}\right\}
$$

which can then be subjected to limit constraints. The result of the nonlinear solution shown in Fig. 5 for both canard-fixed and controlled cases. After convergence to a steady-state solution, an accuracylimited time step size of $46 \mu s$ was used, with a computational cost of five Cray Y-MP CPU hours. Grid communication was approximately one-third of the overall CPU time. Figure 5 shows similar behavior to the linearized cases, again with a modestly growing envelope for the canard-fixed case. and damped oscillations for the controlled case. This comparison provides a degree of validation of the implementation of the control law in the coupled code.

\section{2-D Resonating Cavity}

In order to establish confidence in the numerical method, a two-dimensional cavity computation was undertaken to demonstrate and validate self-induced cavity resonance. Details of this and threedimensional cavity computations may be found elsewhere. ${ }^{\top}$

The test conditions, specified to match experiment, ${ }^{22}$ were

$$
\begin{array}{lll}
M_{\infty}=0.9, & R e_{L}=1.47 \times 10^{6}, \quad L=8 \mathrm{in} . \\
\rho_{\infty}=0.40 \mathrm{~kg} / \mathrm{m}^{3}, & p_{\infty}=2.9 \times 10^{4} \mathrm{~N} / \mathrm{m}^{2}
\end{array}
$$



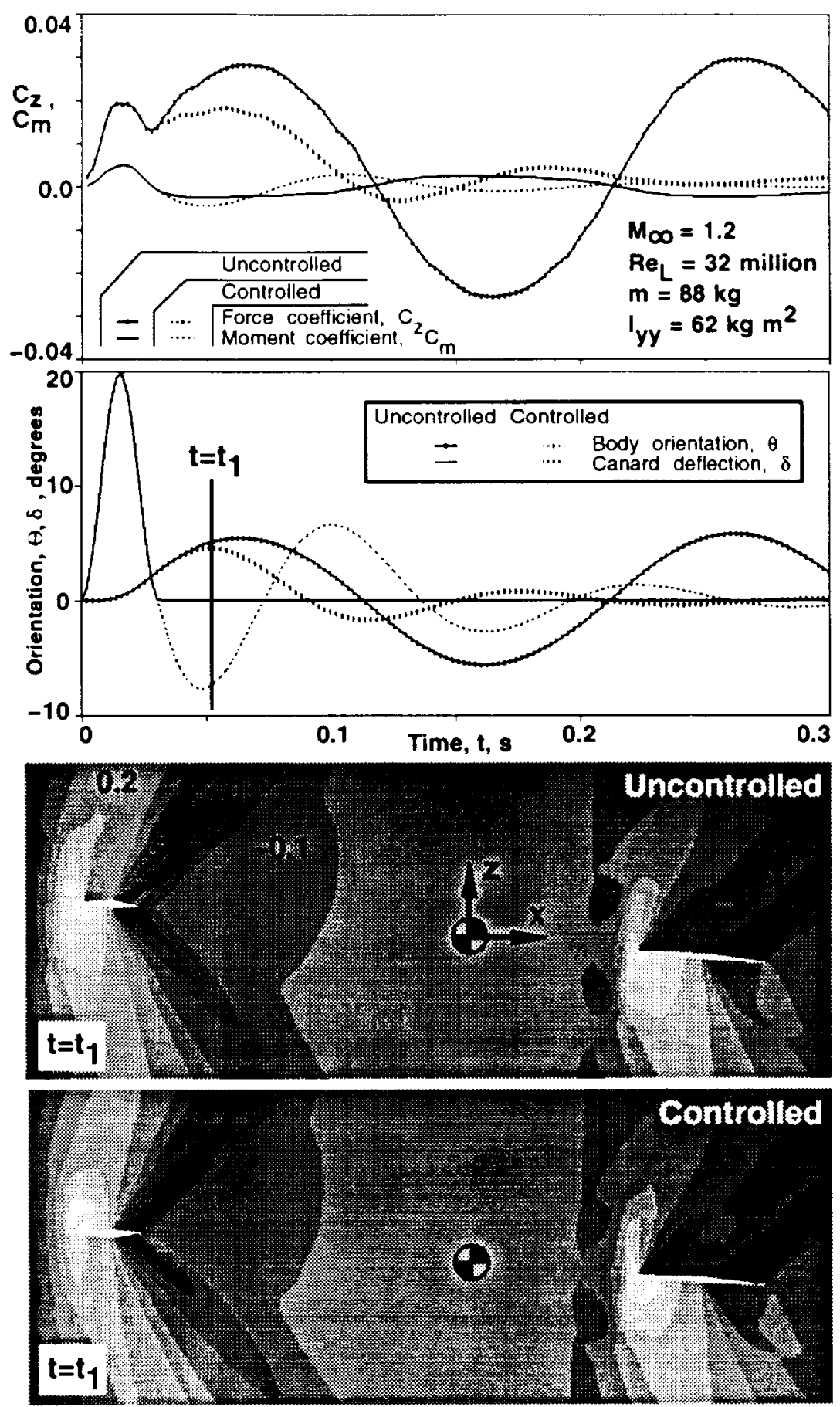

Fig. 5: 2-D missile: Body orientation and canard deflection time histories and instantaneous $C_{p}$ contours for both uncontrolled and controlled cases at $t=t_{1}$ 


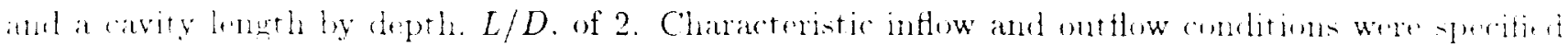

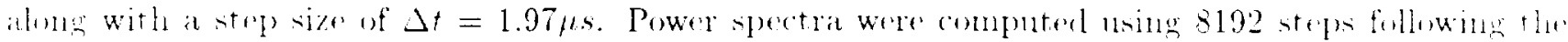
dissipation of the initial transients.

Inspretion of the conpunted cavity pressure history, shown in Fig. 6a. confirms the idealized fredlatck
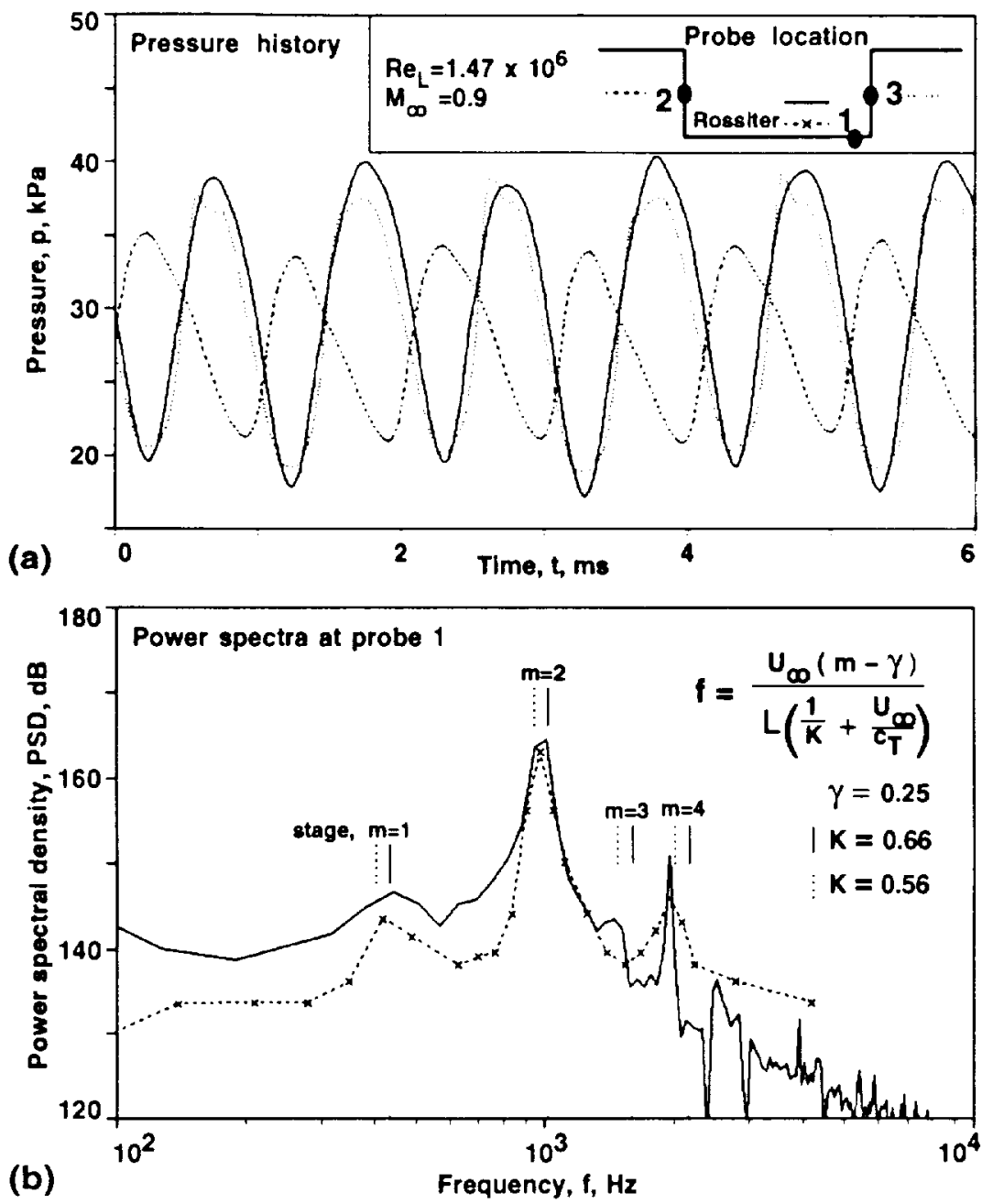

Fig. 6: 2-d cavity: (a) pressure history and (b) power spectra comparison

mechanism identified from Rossiter's experiments. Briefly, the cycle begins with the propagation of an acoustic wave from the aft wall of the cavity to the forward bulkhead. Wave reflection from the forward wall causes the shear layer to bow outwards, shedding vorticity. The deflected shear layer convects downstream and induces another cycle. This coupling of the acoustic and vortical fields is quantified by Rossiter`s empirical model, given in Fig. 6b, which gives only feedback frequencies.

In the frequency domain, comparison of Rossiter's data to present results indicate agreenent in frequency at the peak magnitudes, as shown in Fig. 6b. Magnitudes are higher for the present case by about $2 \mathrm{~dB}$. which can be explained from dimensionality arguments. The solution was also found to be insensitive to second-order dissipation levels within the range 0.3 to 0.5 . Figure $6 \mathrm{~b}$ also shows the resonant modes predicted by Rossiter's equation, showing that $K=0.56$ gives better prediction of the higher modes. Finally, the vertical knife edge schlieren images of Fig. 7 show the qualitative agreement between computed and observed ${ }^{23}$ radiation patterns. 

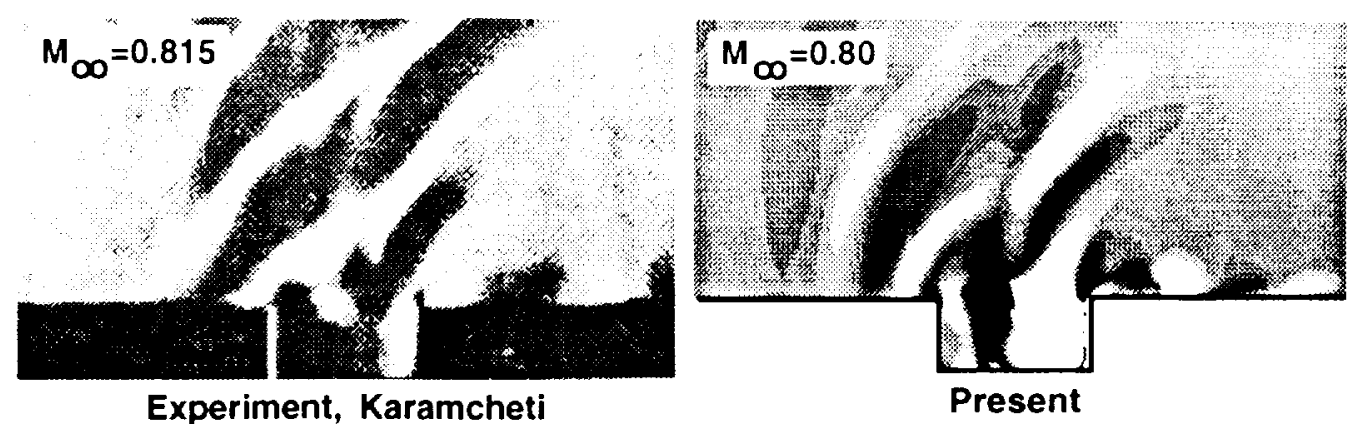

Fig. 7: Comparison of experimental and numerical schlieren images with knife edge vertical

\section{2-D Controlled Store Separation}

Using the control law developed for the 1-DOF simulation above, albeit with an arbitrarily chosen $\theta_{c}=5^{\circ}$, a 3 -DOF simulation of a store separating from a cavity was implemented. The separation began with the application of an $18000 \mathrm{~N}$ ejection force for $0.044 \mathrm{~s}$, with corresponding acceleration of about $20 \mathrm{~g}$, during which the controller was off and no angular velocity was imparted to the store. The solution was initialized with the store fixed in carriage position, allowing damping of the starting numerical transients. Following convergence of the cavity acoustic envelope, the same accuracy-limiter time step size of $46 \mu s$ was used. The time step size was chosen such that the streamwise Courant number was about unity in the shear layer. This restriction is equivalent to allowing an acoustic wave to propagate only one cell in a single step. Ten grids were used for this $1.3 \times 10^{5}$ point domain. The result, shown in Figs. 8 and 9, shows that the nose of controlled store remains pointed away from the parent body, while the canard-fixed store is pointed towards the parent $0.3 \mathrm{~s}$ after release. Since the controlled store is commanded to point away from the parent, the separation is faster for the conl: .lled case than for the canard-fixed store. Inspection of the normal force history shows a component of about. $50 \mathrm{~Hz}$. corresponding to the second stage of Rossiter`s formula. ${ }^{9}$

\section{3-D Missile Stability Derivatives}

In order to determine the proper feedback gains. the stability derivatives of the missile must be computed. For this 3-DOF simulation this includes $C_{m_{\alpha}}, C_{m_{\delta_{c}}}$, and $C_{m_{q}}$. These paraneters were computed from four cases: using a nominal $\theta=\delta_{c}=0$, a constant pitch attitude $\theta=0.04 \mathrm{rad}$. $\delta_{c}=0$. a constant deflection angle $\theta=0, \delta_{c}=0.04 \mathrm{rad}$, and a constant pitch rate $\dot{\theta}=9 \mathrm{rad} / \mathrm{s}$. Canard motion was permitted by the $\frac{1}{16}$ in. nominal gap between the missile body and canard. This is a similar arrangement to the actual missile, albeit without the connector pin in the numerical model. The force and moment history was converged three orders of magnitude, approximately 2000 steps. on this 18 grid solution containing approximately 1.5 million points. Figure 10 shows the geometry used for this portion of the study, along with coefficient of pressure, $C_{p}$, contours.

\section{3-D Cavity Store Separation}

The geometry, shown in carriage position, can be seen in Fig. 11. The domain contains about 2.2 million points distributed in 25 grids, with the missile grids being re-used from the previous stability derivative study. An example of the initialization of the cavity store separation problem is shown in Fig. 12. This store-fixed simulation will be run approximately five characteristic times, until the artificial starting transients have dissipated, after which ejection will begin. The ejection forces applied to the store will be such that the velocity at the end of the $8 \mathrm{in}$. piston stroke was $30 \mathrm{ft} / \mathrm{s}$ normal to 

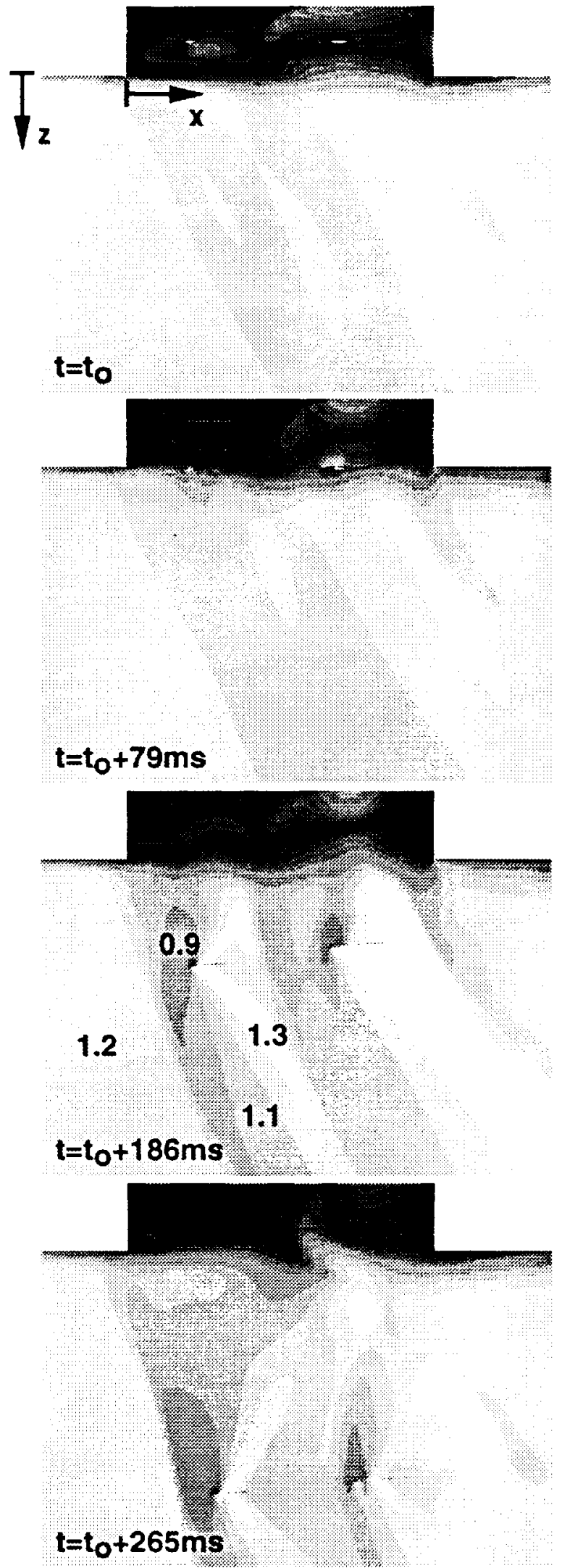

Fig. 8: 2-D controlled store separation: instantaneous Mach contours 


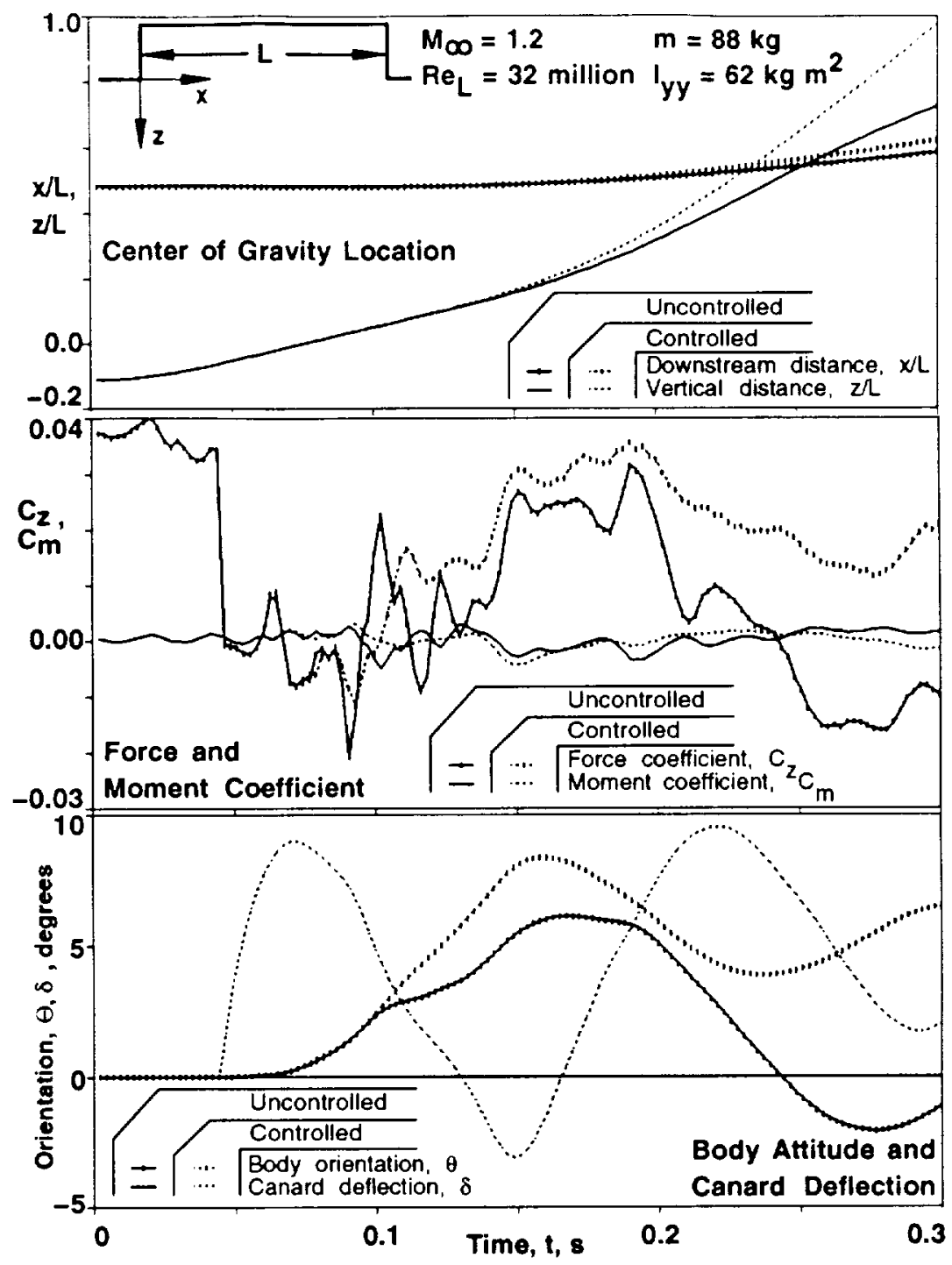

Fig. 9: 2-D controlled store separation: time histories of a) center of gravity, b) force and moment coefficients, and c) body attitude and canard deflection angles

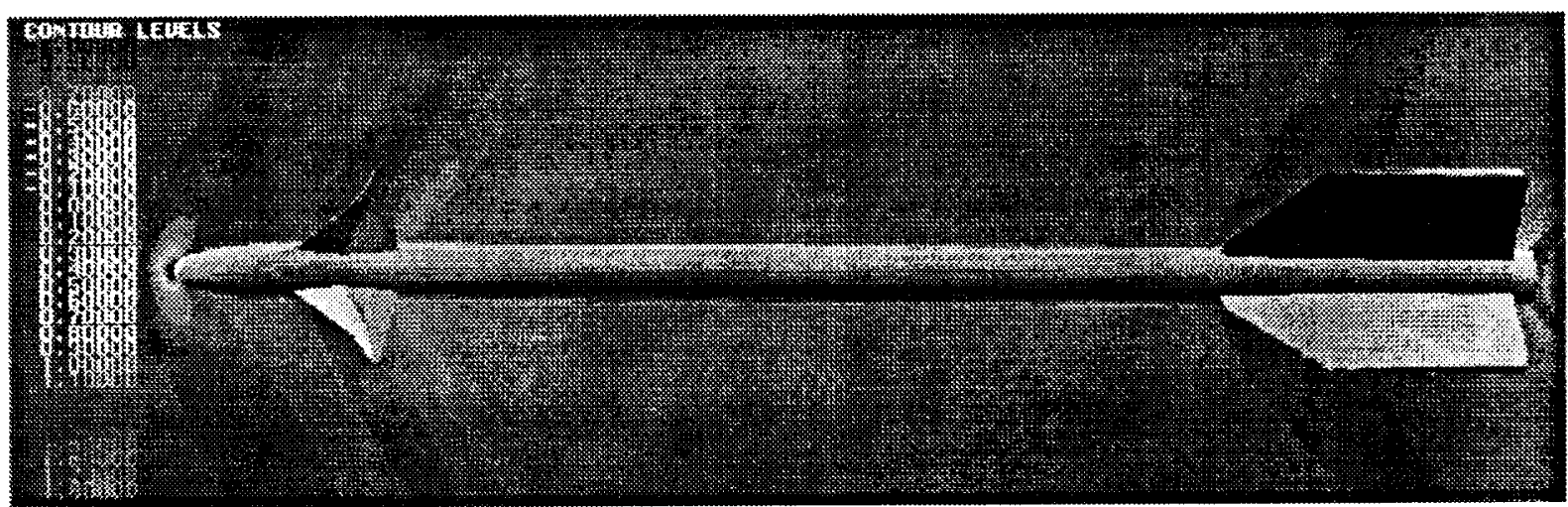

Fig. 10: 3-D missile: $C_{p}$ contours 


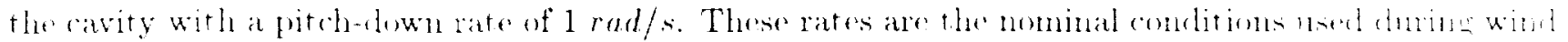
thinled trinting.

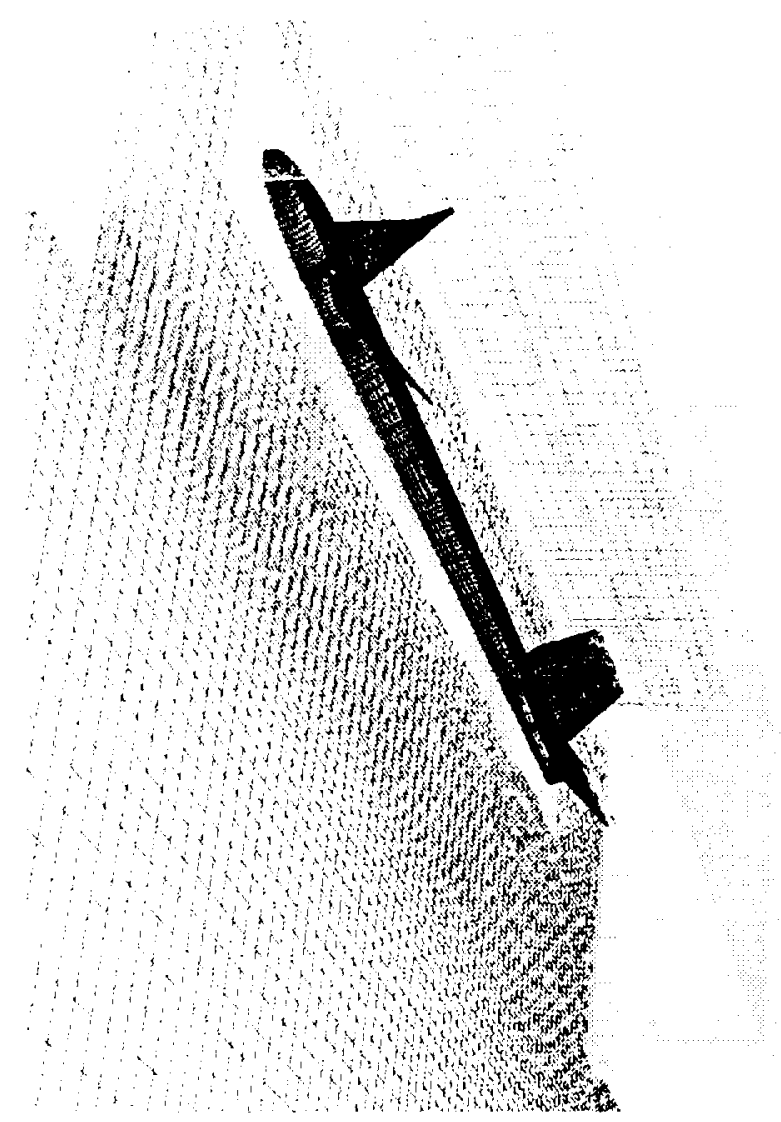

Fig. 11: 3-D store separation: grids for missile in carriage

\section{Conclusions}

A pitch attitude control law was implemented in a coupled Navier-Stokes/rigid-body dynanics code. Comparison of nonlinear with linearized results showed reasonable comparison for the perturbed case with the controller active or off. Application of the control law to a two-dimensional, three-degree-offreedom cavity store separation revealed improved trajectory characteristics. The generalized coding of aerodynamic effector kinematics in the coupled code will allow rapid implementation of existing control laws. Simulation of the coupled nonlinear aircraft trajectory can then be used to computationally prototype the control system.

\section{Note to the Reviewer}

Computation of a three-dimensional canard-fixed store separating from a cavity is in progress and will be compared to the experimental data of Dix and Dobson. ${ }^{6}$ Comparison of the numerical and experimental results will provide an additional measure of validation. After completion of the uncontrolled case, a simulation of the pitch-controlled case will commence. Determination of the feedback gains for this three-dimensional simulation will require the computation of the stability derivatives. which will also be accomplished via the Navier-Stokes equations. Assessment of the uncontrolled and controlled cases will show if improved cavity store separation characteristics can be achieved via canard effectors. 


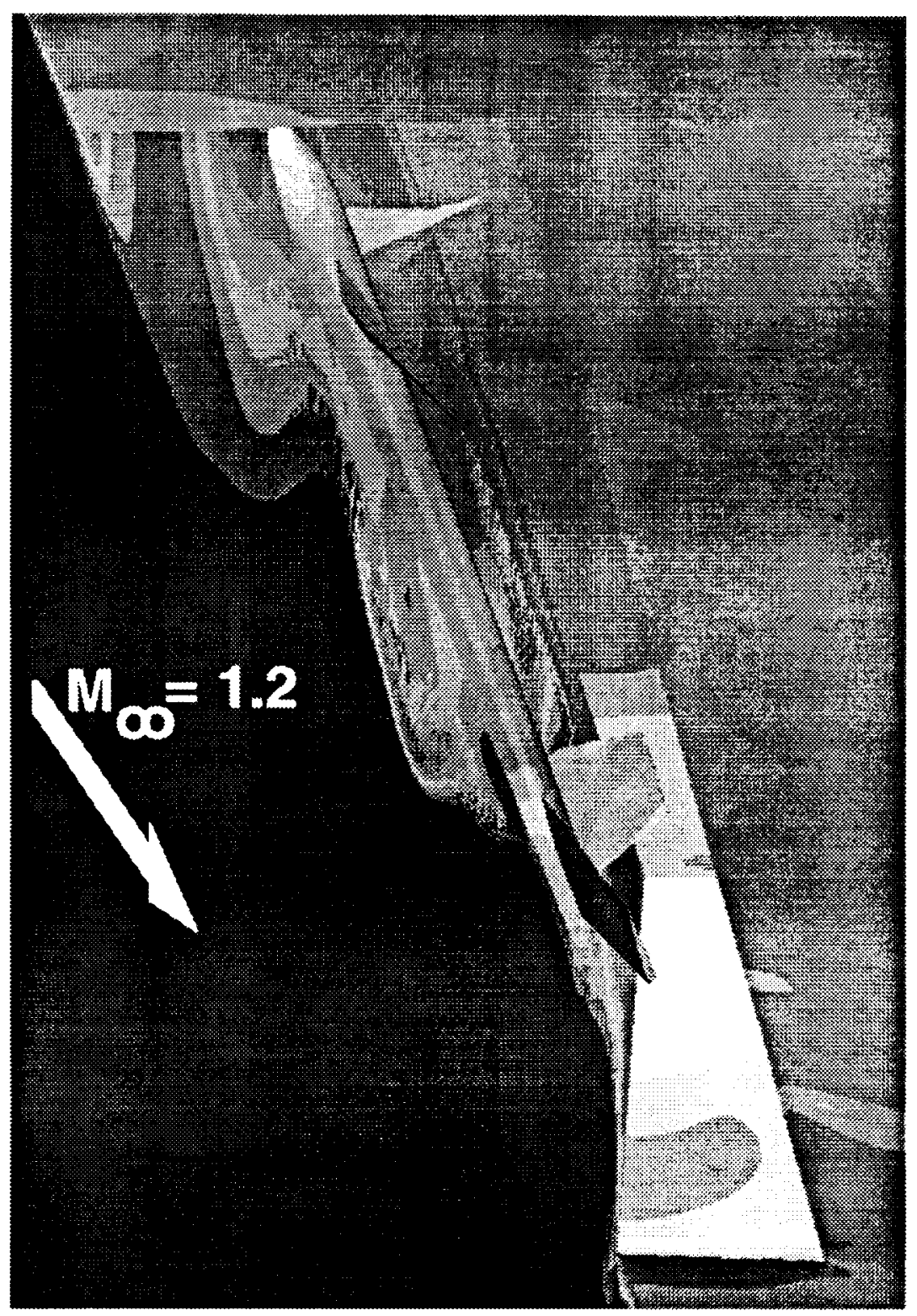

Fig. 12: 3-D store in carriage: instantaneous contours of $\rho u$ on the symmetry plane and $C_{p}$ contours on the wetted surfaces 


\section{Acknowledgements}

This investigation was partially supported by grant NCC-2-677. Computations were performed on the Numerical Aerodymanic Simmlator (NAS) Cray Y-MP and C90, the NASA/Ames Central Conputing Facility (CCF) Cray Y-MP. and a Silicon Graphics 4D35TG workstation at the Computational Aerosciences Branch. Special thanks is owed to Richard E. Dix. and Drs. Robert L. Meakin. Kalpana Chawla. George Meyer, and James A. Franklin for their assistance with the experimental data. software tools, and interpretation of results.

\section{References}

1 Franklin. J.A., Hynes, C.S., Hardy, G.H., Martin. J.L.. and Innis R.C., "Flight Evaluation of Augmented Controls for Approach and Landing of Powered-Lift Aircraft," AIAA J. of Guidance, Control, and Dynamics, Vol. 9, Sep.-Oct. 1986, pp. 555-565.

2 Franklin, J.A.. Stortz, M.W., and Mihalow, J.R., "Integrated Flight/Propulsion Control for Supersonic STOVL Aircraft," Intl. Powered-Lift Conference, London. Aug. 1990. pp. III.9.1-12.

3 Meyer. G.. and Cicolani. L., "Application of Nonlinear System Inverses to Automatic Flight Control Design - System Concepts and Flight Evaluations." AGARDograph No. 251. J11y 1981.

4 Smith, G.A., and Meyer, G.,"Aircraft Automatic Flight Control System with Model Inversion." Control and Dynamics Systems, Vol. 38, Advances in Aeronautical Systems, Academic Press. San Diego, 1990.

5 Tobak, M.. and Schiff. L.B.,"Aerodynamic Mathematical Modeling - Basic Concepts,." AGARD Lecture Series No. 114 on Dynamic Stability Parameters, Lecture No. 1, Mar. 1981.

6 Dix, R.E., and Dobson Jr., T.W., "Database for Internal Store Carriage and Jettison," AEDC-TR90-23. Nov. 1990.

7 Atwood, C.A., and Van Dalsem, W.R., "Flowfield Simulation about the SOFIA Airborne Observatory," AIAA Paper 92-0656, Jan. 1992. AIAA J. of Aircraft, to appear.

8 Suhs, N.E.,"-Transonic Flow Calculations for a Cavity with and without a Store." AEDC-TR-92-4. Sep. 1992.

9 Atwood. C.A., "Selected Computations of Transonic Cavity Flows," Forum on Computational Aeroand Hydro-Acoustics, 1993 ASME Fluids Engineering Conference. Washington D.C.. June 1993.

${ }^{10}$ Meakin, R.L., "Computations of the Unsteady Flow About a Generic Wing/Pylon/Finned-Store Configuration," AIAA Paper 92-4568. Aug. 1992.

11 Lijewski, L., and Suhs, N.E., "Chimera-Eagle Store Separation," AIAA Paper 92-4569, A11g. 1992.

12 Löhner, R.. "Three-Dimensional Fluid-Structure Interaction Using a Finite Element Solver and Adaptive Remeshing," Computing Systems in Eng.. 1. Nos. 2-4, 1990. pp. 257-272.

13 Pulliam, T.H. and Chaussee, D.S., "A Diagonal Form of an Implicit Approximate-Factorization Algorithm," J. Comp. Phys., Vol. 39, Feb. 1981, pp. 347-363.

14 Benek, J.A., Buning, P.G., and Steger, J.L., "A 3-D Chimera Grid Embedding Technique." AIAA Paper 85-1523, July 1985. 
15 Baldwin. B.S. and Lomax. H.. "Thin-Layer Approximation and Algebrair Morled for Sepratent Turbulnut Flows." AIA Piper 78-257. Jan. 1978.

16 Buning. P.G. and Chan. W.M.. "OVERfloW/ F3D User's Manual. Version 1.5." NASA/ARC. Nov. 1990.

17 Steinbrenner. J.P., Chawner. J.R., and Fonts. C.L., "A Structured Approach to Interactive Multiple Block Grid Generation." AGARD FDP Specialists Mtg. on Mesh Generation for Complex ThreeDimensional Configurations, Loen. Norway, May 1989.

18 Chan. W.M. and Steger, J.L., "Enhancements of a Three-Dimensional Hyperbolic Grid Generation Scheme," App. Math. and Comp., Vol. 51, pp. 181-205.

19 Meakin. R.L., "A New Method for Establishing Inter-Grid Communication Among Systems of Overset Grids," AIAA Paper 91-1586, June 1991.

20 McCann, K.M., and Meakin. R.L.,"GDCF - An Interactive Approach to Domain Connectivity Among Systems of Overset Grids." AIAA Technical Note, AIAA 11th Computational Fluid Dynamics Conference, Orlando, FL. July 1993.

21 Meakin, R.L., "Overset Grid Methods for Aerodynamic Simulation of Bodies in Relative Motion." 8th Aircraft/Stores Compatibility Symposium, Oct. 1990.

22 Rossiter, J.E., "Wind-Tunnel Experiments on the Flow Over Rectangular Cavities at Subsonic and Transonic Speeds," Royal Aircraft Establishment Reports and Memoranda No. 3438, Oct. 1964.

23 Karamcheti, K., "Acoustic Radiation from Two-Dimensional Rectangular Cutouts in Acrodynamic Surfaces," NACA TN-3487, 1955. 